\title{
Gestation-specific reference intervals for fetal cardiac Doppler indices from 12 to 40 weeks of gestation
}

\author{
Rajeswari Parasuraman ${ }^{1}$, Clive Osmond $^{2}$, David T. Howe ${ }^{1}$ \\ ${ }^{1}$ Wessex Maternal and Fetal Medicine unit, Princess Anne Hospital, Southampton, UK \\ ${ }^{2}$ MRC Lifecourse Epidemiology Unit, Southampton General Hospital, Southampton, UK \\ Email: rajeswari.parasuraman@uhs.nhs.uk
}

Received 17 September 2012; revised 16 October 2012; accepted 25 October 2012

\begin{abstract}
We aimed to establish gestation age specific reference intervals for Doppler indices of fetal cardiac function from 12 to 40 weeks of pregnancy. In a cross-sectional observational study of singleton pregnancies, examinations were performed in 221 women evenly distributed across each week of pregnancy. Blood flow through the four cardiac valves was examined with Doppler. For the atrioventricular valves, velocity and duration of early (E) and atrial (A) waves and the interval (a) between $\mathrm{E} / \mathrm{A}$ complexes was recorded. For the outflow valves, the duration (b), peak and average velocity of flow in systole was measured. Myocardial performance index (MPI) was calculated as $(\mathbf{a}-\mathbf{b}) / \mathbf{b}$. Outlet valve diameters were measured and cardiac outputs were calculated. Gestation age specific ranges were constructed for all these parameters. We demonstrated that the cardiac output, peak systolic and time-averaged velocity increase with advancing gestation. However the MPI and E/A ratios show little change across gestation. Fetal cardiac physiology can be studied and Doppler indices reliably measured as early as the late first trimester of pregnancy. Establishing gestation age specific ranges for various cardiac indices throughout pregnancy will help the study of development of fetal cardiac function.
\end{abstract}

Keywords: Cardiac Doppler; Fetal; Gestation Age; Reference Range; Ultrasound

\section{INTRODUCTION}

Doppler ultrasound has been used for some years to assess fetal cardiac function including assessment of cardiac output and measures of diastolic function including assessment of the E/A ratio - the ratio of early diastolic peak flow through the atrioventricular valves, to the peak with atrial contraction. Recently there has been particular interest in the myocardial performance index (MPI) as an assessment of both diastolic and systolic cardiac function. The MPI was first reported by Tei to be useful in determining myocardial performance in adults with amyloidosis [1]. The Tei index has been used in assessment of children with congenital heart disease [2] and also in the prediction of onset of twin to twin transfusion in monochorionic twin pregnancies [3]. They have also described an increase in the index in large for gestational age fetuses of diabetic mothers during the latter half of pregnancy. They proposed that this may be due to abnormal myocardial function predisposing to cardiomyopathy in later life. An increase in the modified MPI with increaseing cardiac dysfunction associated with fetal growth restriction has been described [4].

The potential clinical applications of MPI and other measures of fetal cardiac function are still being evaluated. We are interested in studying the development of cardiac function during pregnancy because of its possible relationship with adult cardiac physiology and disease. The concept of developmental origins of adult disease is well established. Changes in growth in early development increase the susceptibility to disease many years later [5]. Animal studies have shown mechanisms by which adverse intrauterine environments may be linked to adult cardiac disease. The developing heart grows by a process of myocyte division, but when mature cell division ceases further growth is by cell hypertrophy. The number of capillaries in mature myocardium is proportionate to the number of cells, so that if the number of myocytes is reduced, but each cell is larger, the distance between capillaries is increased, potentially increasing sensitivity to ischaemia [6]. In a study of experimentally induced intra-uterine growth restriction in rats, evidence of left ventricular remodelling, including altered myocyte volume and reduced contractility has been demonstrated in response to constant voltage stimulation [7].

We hypothesise that the structural changes that lead to later heart disease may be associated with demonstrable changes in fetal cardiac function during pregnancy. To form the basis of future studies of the factors affecting 
the development of fetal cardiac function we aimed to establish gestation-specific reference ranges for Doppler parameters of cardiac function for both the right and left sides of the heart from 12 to 40 weeks of pregnancy. By measuring the timing and velocity of flow we could construct gestation specific reference intervals of both directly measured and calculated indicators of cardiac function. The majority of these measures have been studied before by various authors, although, with the exception of cardiac output [8] none have studied the parameters throughout pregnancy from 12 weeks till term.

\section{MATERIALS AND METHODS}

This was a cross-sectional observation study of women undergoing normal singleton pregnancy. When women attended the ultrasound department for routine dating or anomaly scans they were invited to take part in the study by coming for an additional research scan. 275 women initially agreed to take part, of whom 224 attended for their research scan. The scans were arranged so that each woman attended for a single assessment of fetal cardiac function and approximately 8 women were recruited for each week of pregnancy from 12 to 40 weeks. We obtained statistical advice before beginning the study to calculate how many women needed to be recruited. The method used to calculate the centile curves meant that the estimate of the median at a particular week of gestation was not based solely on the observations made in that week. The curve was made to fit smoothly over the entire course of gestation, and hence borrowed strength from neighbouring gestational weeks. The study was approved by the local research ethics committee.

The exclusion criteria included women who developed complications of pregnancy prior to their scan such as pre-eclampsia, cholestasis or intra-uterine growth restricttion (defined as a estimated weight below the $5^{\text {th }}$ centile for gestation) and women whose fetus was found to have a significant structural anomaly (defined as one which would normally require onward referral to the fetal medicine unit for assessment and management).

Two-dimensional fetal echocardiography was performed using a Toshiba Aplio ultrasound machine. Examination of blood flow through the right heart was made by Doppler assessment of the timing and velocity of flow through the tricuspid and pulmonary valves. In order to make the measurements, the Doppler gate was reduced to its minimum size $(1 \mathrm{~mm})$ and placed centrally on the outlet of the tricuspid valve, adjacent to the tips of the valve in diastole, so that the valve movements are detectable. For the pulmonary outflow the gate was placed just beyond the valve. The valves "clicks" are evident in the Doppler flow and help to define the opening and closing of the valves. The ultrasound probe was placed so that the beam was as near to parallel as possible with the direction of blood flow and always less than 30 degrees. For velocity measurements the machine settings were used to correct for the angle of insonation if the beam was not truly parallel.

There are two phases of flow through the tricuspid valve: the $\mathrm{E}$ wave resulting from early, passive flow as the heart relaxes in diastole and the A wave from active filling as the atria contract. The duration and velocity of these peaks was measured. The duration of the $\mathrm{E}$ wave and of the E/A complex was measured along with the tricuspid closing to opening time ("a" in Figure 1) and the E/A ratio was also assessed To measure the "a" we placed the calipers just within the EA complexes so that the outer border of the caliper line was just abutting the complex.

There is a single peak of flow through the pulmonary valve, during cardiac systole and the peak and average velocity of systolic flow and length of the ejection time (b) was measured (Figure 2). We placed the caliper lines so that the inner borders were touching the ejectionwaveform. The stroke volume was calculated as the time

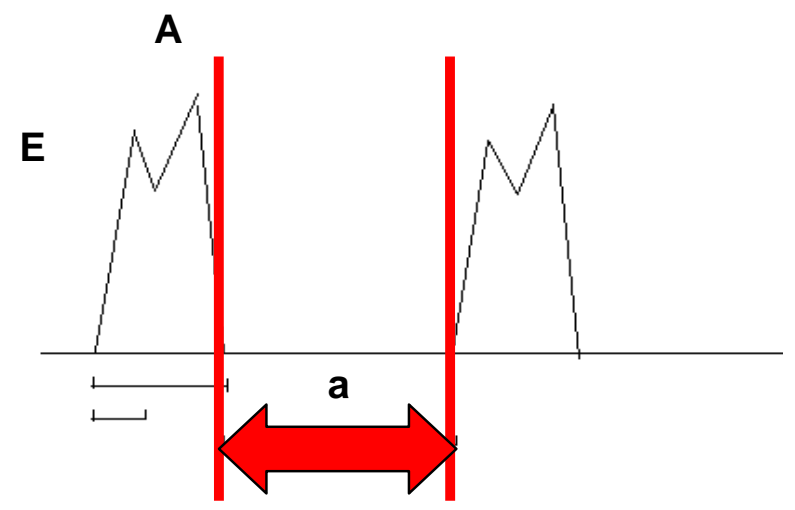

(a)

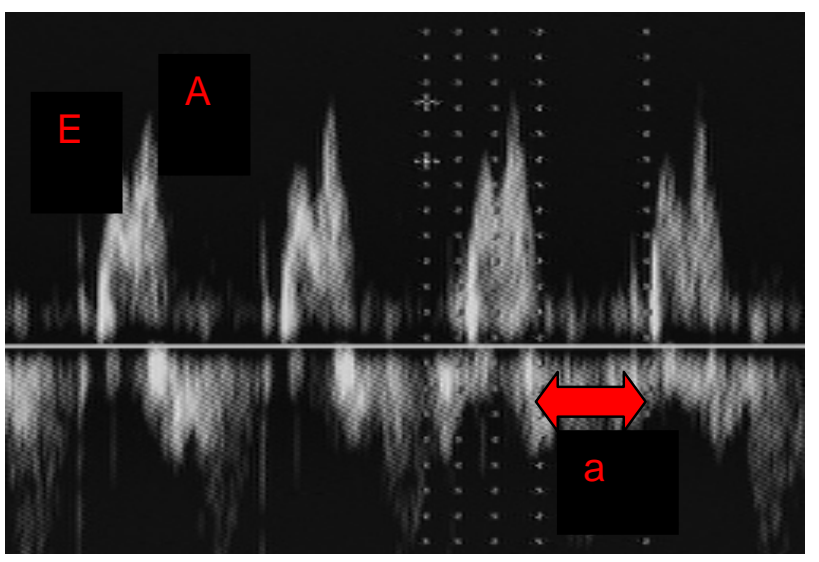

(b)

Figure 1. (a) Schematic diagram of atrioventricular waveform showing the $\mathrm{E}$ and $\mathrm{A}$ waves, and the time between complexes "a" used to calculate MPI as described in the methods; (b) Pulsed Doppler image of atrioventricular waveform. 


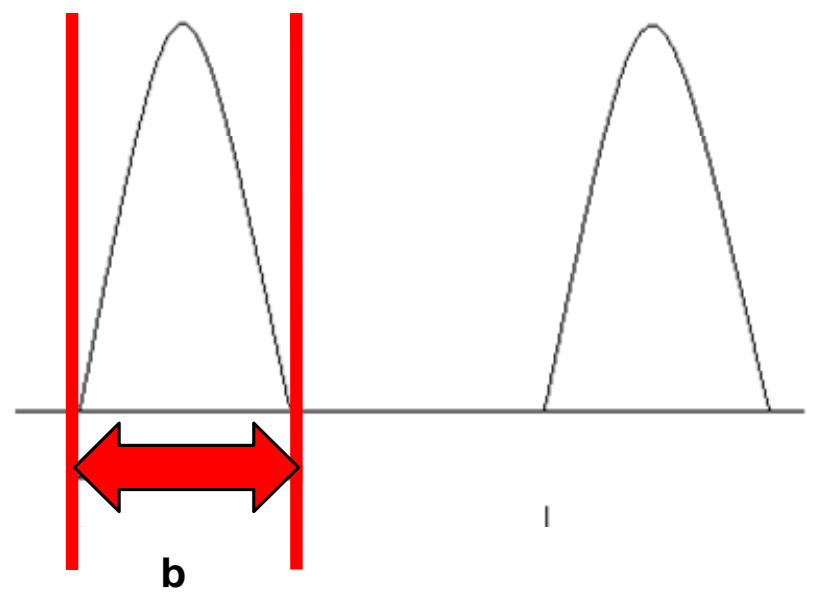

(a)

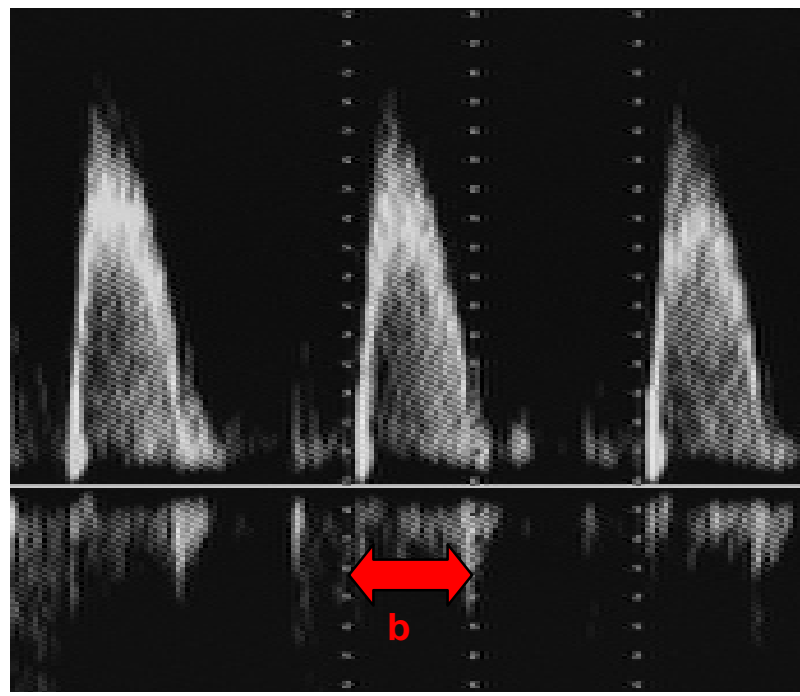

(b)

Figure 2. (a) Schematic diagram of outlet valve waveform demonstrating length of the ejection time, "b" used to calculate the MPI as described in the methods; (b) Pulsed Doppler image of outlet valve waveform.

averaged velocity $\mathrm{x}$ output time $\mathrm{x}$ cross-sectional area of the outlet valve, and the cardiac output as the stroke volume $\mathrm{x}$ heart rate. To measure the pulmonary valve diameter, the ultrasound beam was placed perpendicular to the valve to define the vessel borders clearly and the diameter measured three times, with the average value used for the calculations. The cross-sectional area of the outlet valve was calculated from the average measured diameter.

The myocardial performance index (MPI) was calculated as $(a-b) / b$. We used the method originally described by Tei, with separate acquisition of the waveforms at the atrioventricular valves and the outflow valves. This method was chosen because we wanted to evaluate both sides of the heart and to make measurements at early gestations. The method described by Friedman to calculate MPI using simultaneous viewing of the movements of the aortic and mitral valves for the left ventricle [9] is not feasible for the right heart due to the anatomical separation of the pulmonary and tricuspid valves nor is it practical in early pregnancy due to the small size of the valves at that gestation.

Examination of blood flow through the left heart was made using the same techniques to assess the flow through the mitral and aortic valves. The same measurements were made as for the right heart. For each measurement we used the average values from three consecutive Doppler complexes.

To assess the reproducibility of measurements we repeated all the measurements from each valve in 61 women. In 19 women, the measurements were made by two operators DTH and RP to assess inter-observer variability and in 42 the measurements were repeated by the same operator for intra-observer variation.

\section{RESULTS}

224 women attended for a research scan. Three women were excluded after a structural/chromosomal abnormality was identified in the fetus (one with aortic stenosis, one with rhabdomyomas and one with trisomy 21) so complete data was obtained in 221 women. The fetus that developed aortic stenosis was scanned at 26 weeks, the fetus with rhabdomyomas was scanned at 18 weeks and fetus with trisomy 21 was scanned at 17 weeks. After the exclusions, we had 9 women at 18 weeks included for the data and 7 each at 17 and 26 weeks. As previously mentioned the curve borrowed strength from neighbouring gestational weeks and the estimate of the median at a particular week of gestation was not based solely on the observations made in that week retaining the validity of our centile curves despite the exclusions. We checked that there were no additional late diagnoses of cardiac abnormalities within a year of birth by cross-checking all the babies with the regional congenital anomaly database, which includes all major congenital cardiac abnormalities found after birth. We estimated the gestational mean of a fetal Doppler measurement using a cubic polynomial in gestational age. We then modelled the absolute residual as a linear function of gestational age (though a quadratic function was needed for the two cardiac output variables) in order to estimate the standard deviation of the fetal measurement. We combined these estimates to generate the reference interval graphs, checking for outliers and symmetry of the distributions at each gestational age. The distribution of the cardiac output variables was right-skewed and hence we log transformed them for analysis. All fetuses had biometric measurements within the normal range for gestation at the time of the scan.

The intra-observer reproducibility of the MPI and E/A 
ratios were analysed in 42 women between 20 to 38 weeks gestation. Mean left ventricular (LV) MPI was 0.346 with a standard deviation of 0.142 and the mean right ventricular (RV) MPI was 0.405 with a standard deviation of 0.153 . For repeated assessment of MPI, the mean difference for LV MPI was -0.03 (95\% limits of agreement [LA], -0.281 to 0.275 ) and for RV MPI was -0.02 ( $95 \%$ LA, -0.32 to 0.28 ). Mean E/A ratio for the left heart was 0.68 with standard deviation of 0.072 and the mean E/A ratio for the right heart was 0.716 with a standard deviation of 0.109 . The mean difference of repeat measurements for E/A ratio for the left heart was $0.01(95 \%$ LA, 0.203 to 0.223$)$ and for the right was -0.01 (95\% LA, -0.151 to 0.131 ).

The cardiac Doppler measurements of 19 subjects were measured by two observers (DTH and RP). The mean (standard deviation) of the differences were: $\mathrm{E} / \mathrm{A}$ ratio left $0.008(0.087) ; \mathrm{E} / \mathrm{A}$ ratio right $0.002(0.068)$; MPI left 0.17 (0.16) and MPI right 0.17 (0.12).

The calculated gestation curves for all these Doppler indices displaying the $3^{\text {rd }}, 10^{\text {th }}, 25^{\text {th }}, 50^{\text {th }}, 75^{\text {th }}, 90^{\text {th }}$ and $97^{\text {th }}$ centiles are illustrated in Figures 3-14. The measured Doppler indices for the left and right heart are shown in parallel. The ventricular inlet measurements are shown in Figures 3 and 4, those for the outlets in Figures 5-10 and the calculated values (cardiac output and myocardial performance index) in Figures 11-14.

The parameters describing the centile lines for each cardiac measurement are shown in Table 1. The mean and spread for each gestational week are described by these parameters as follows:

$$
\begin{gathered}
\text { Mean }=\mathrm{c}_{0}+\left(\mathrm{c}_{1} \times \mathrm{t}\right)+\left(\mathrm{c}_{2} \times \mathrm{t}^{2}\right)+\left(\mathrm{c}_{3} \times \mathrm{t}^{3}\right) \\
\text { Spread }=\mathrm{s}_{0}+\left(\mathrm{s}_{1} \times \mathrm{t}\right)+\left(\mathrm{s}_{2} \times \mathrm{t}^{2}\right)
\end{gathered}
$$

where $\mathrm{t}=($ gestational age in weeks -25$) / 7$.

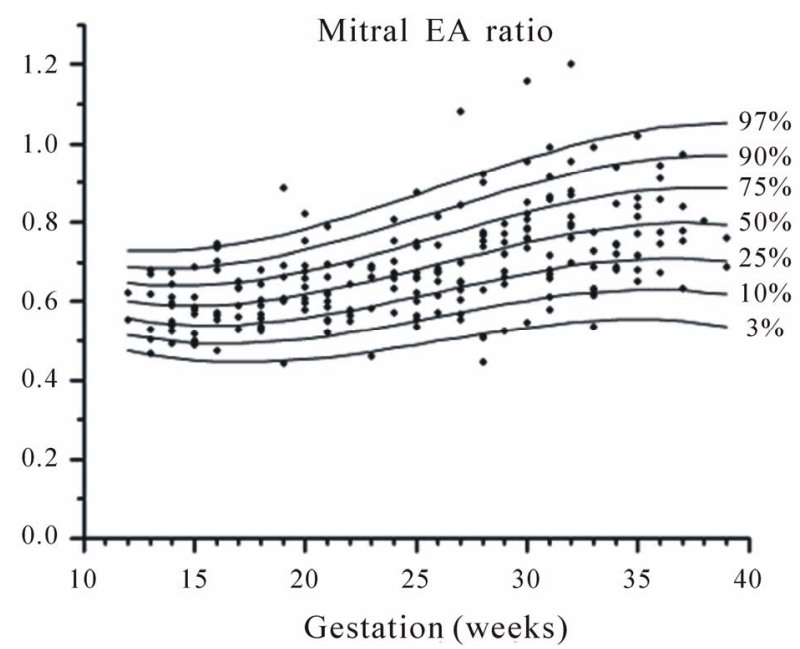

Figure 3. Gestation specific reference intervals for mitral E/A ratio.

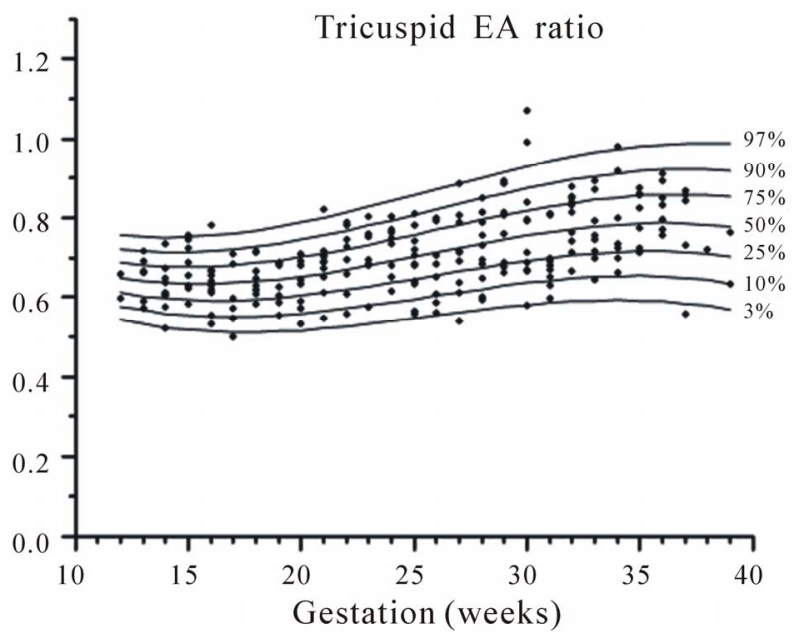

Figure 4. Gestation specific reference intervals for tricuspid E/A ratio.

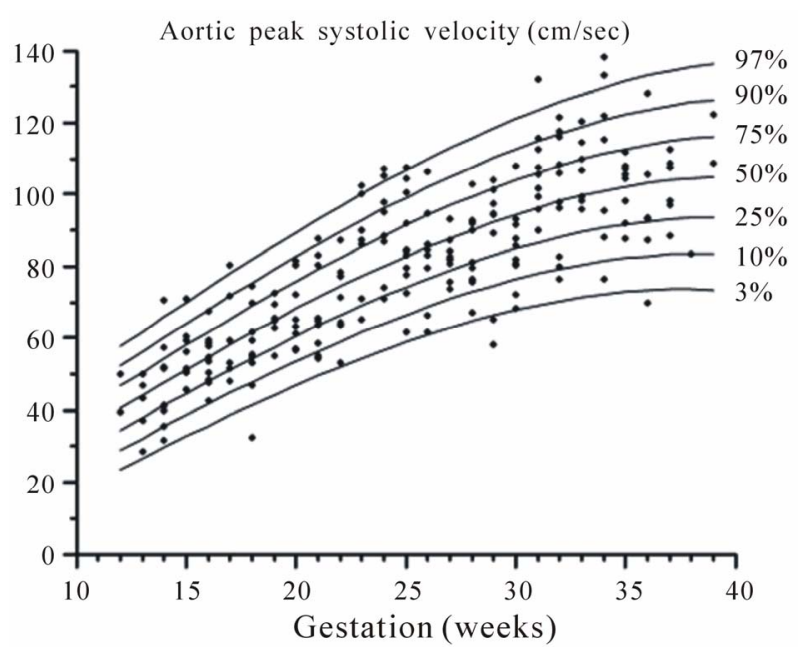

Figure 5. Gestation specific reference intervals for aortic peak systolic velocity.

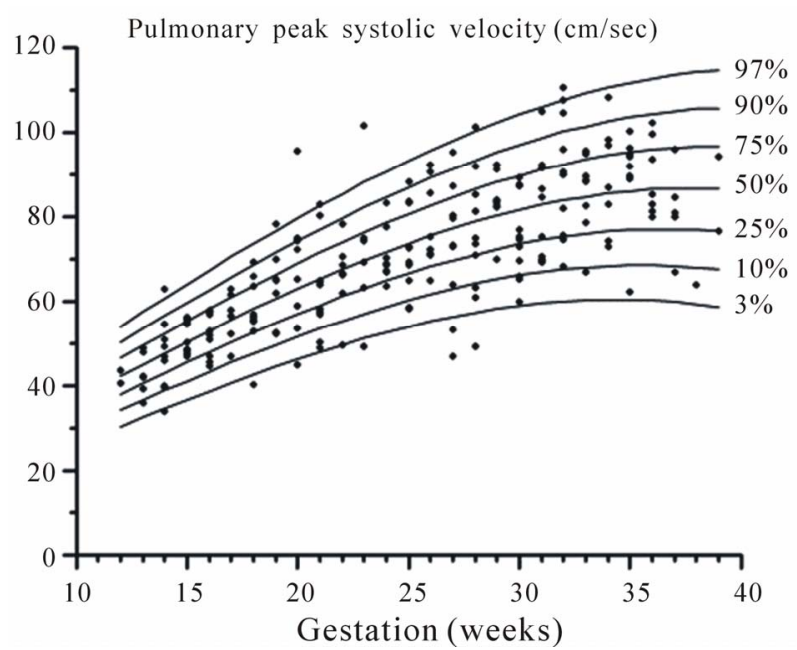

Figure 6. Gestation specific reference intervals for pulmonary peak systolic velocity. 


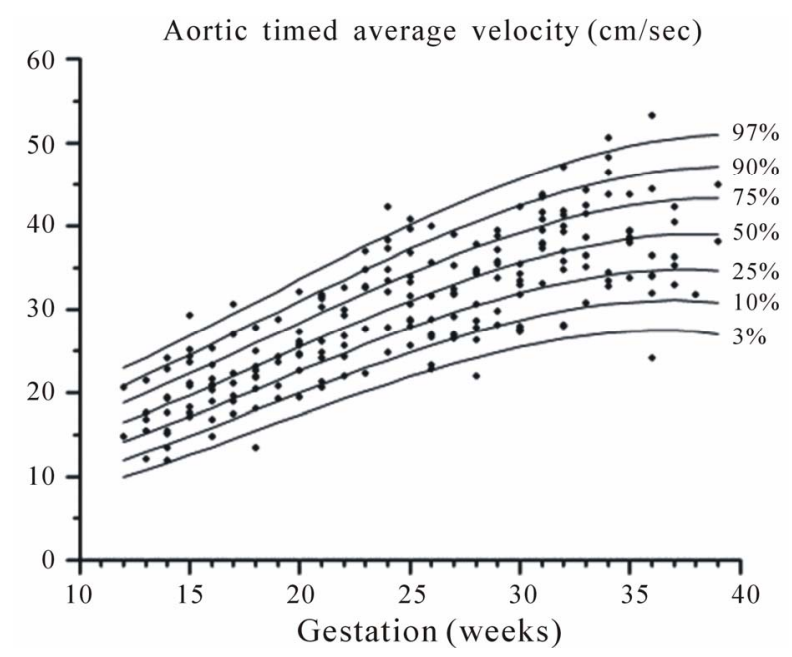

Figure 7. Gestation specific reference intervals for aortic timed average velocity.

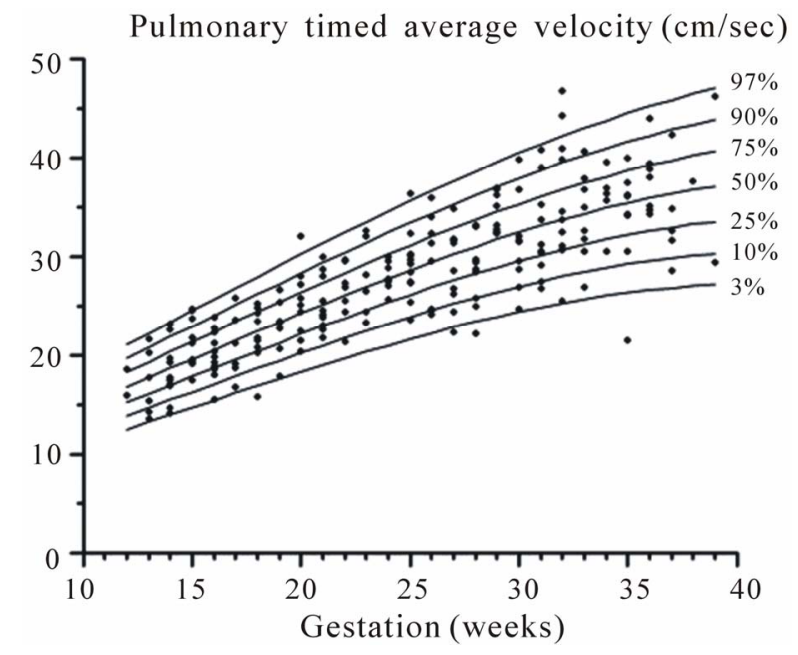

Figure 8. Gestation specific reference intervals for pulmonary timed average velocity.

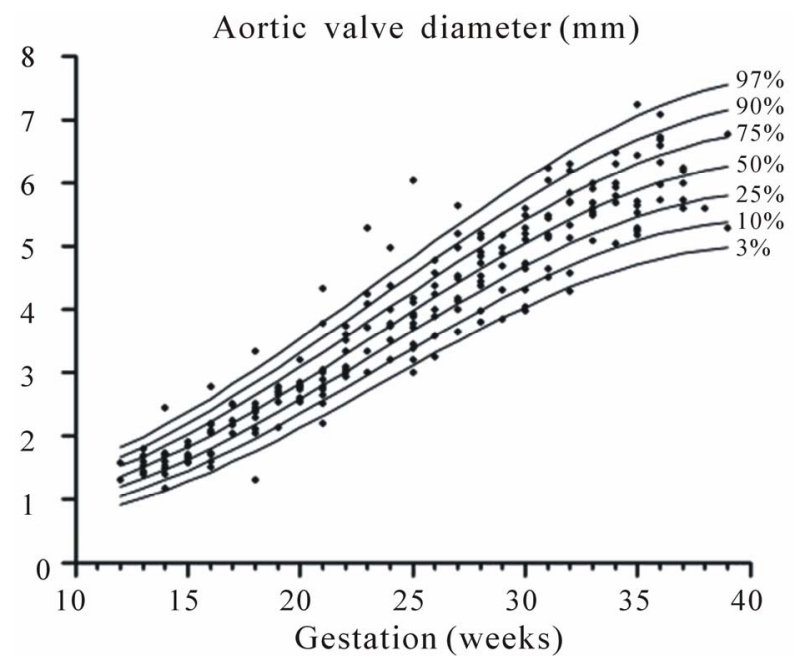

Figure 9. Gestation specific reference intervals for aortic valve diameter.
Pulmonary valve diameter $(\mathrm{mm})$

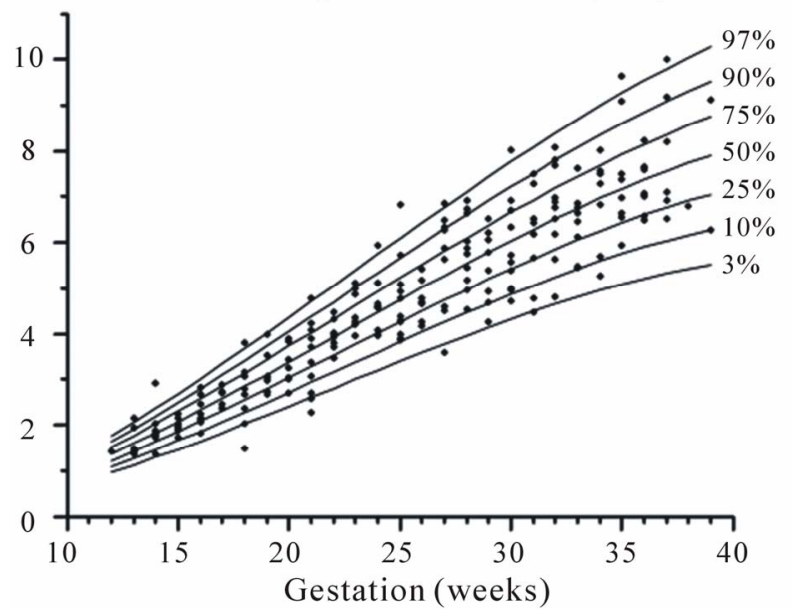

Figure 10. Gestation specific reference intervals for pulmonary valve diameter.

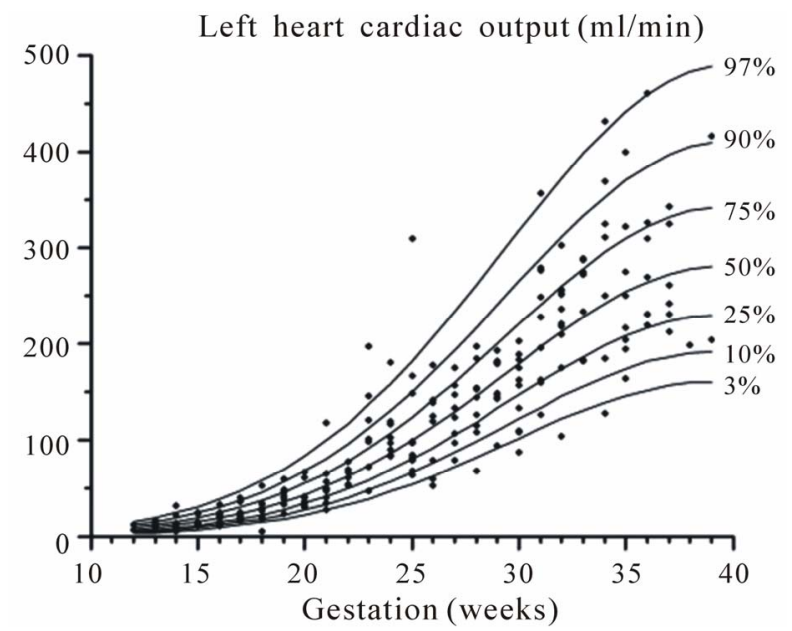

Figure 11. Gestation specific reference intervals for cardiac output of the left heart.

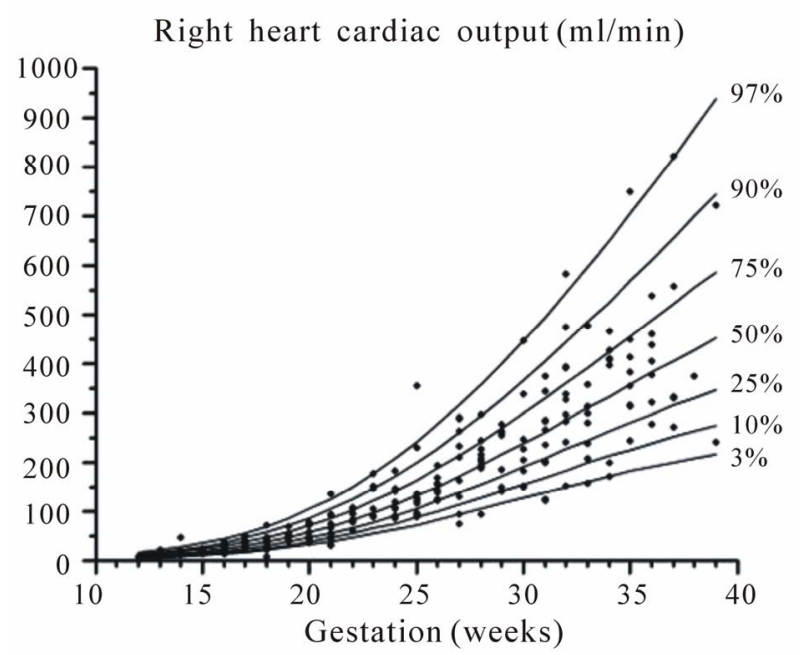

Figure 12. Gestation specific reference intervals for cardiac output of the right heart. 
Left myocardial performance index

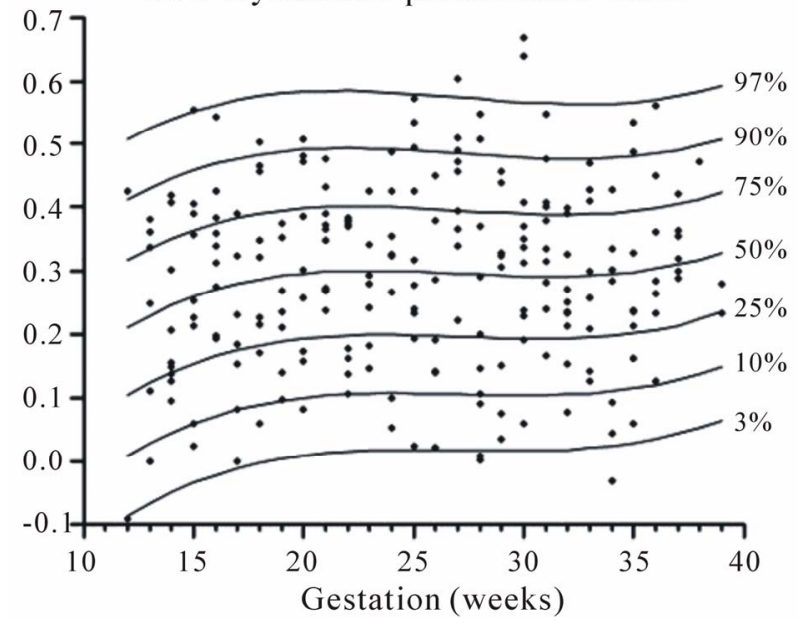

Figure 13. Gestation specific reference intervals for MPI of the left heart.
Right myocardial performance index

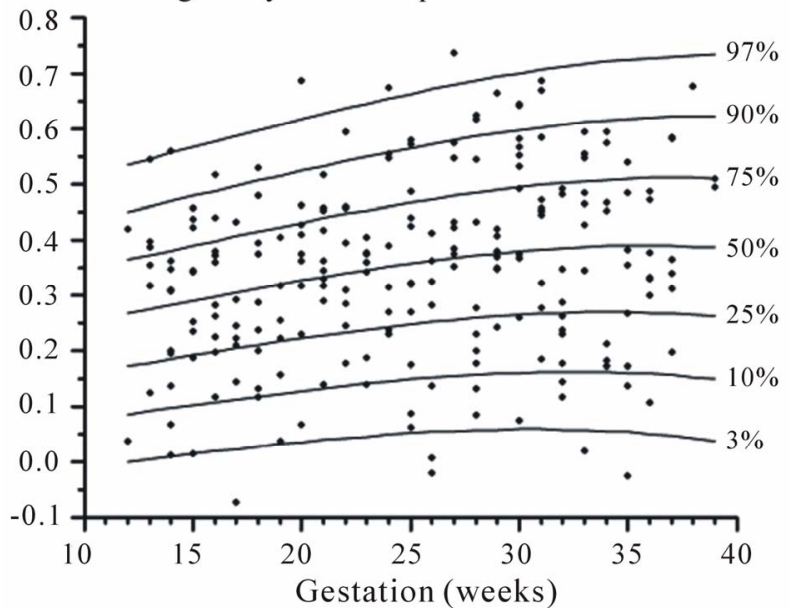

Figure 14. Gestation specific reference intervals for MPI of the right heart.

Table 1. Coefficients used to derive the gestation specific reference ranges.

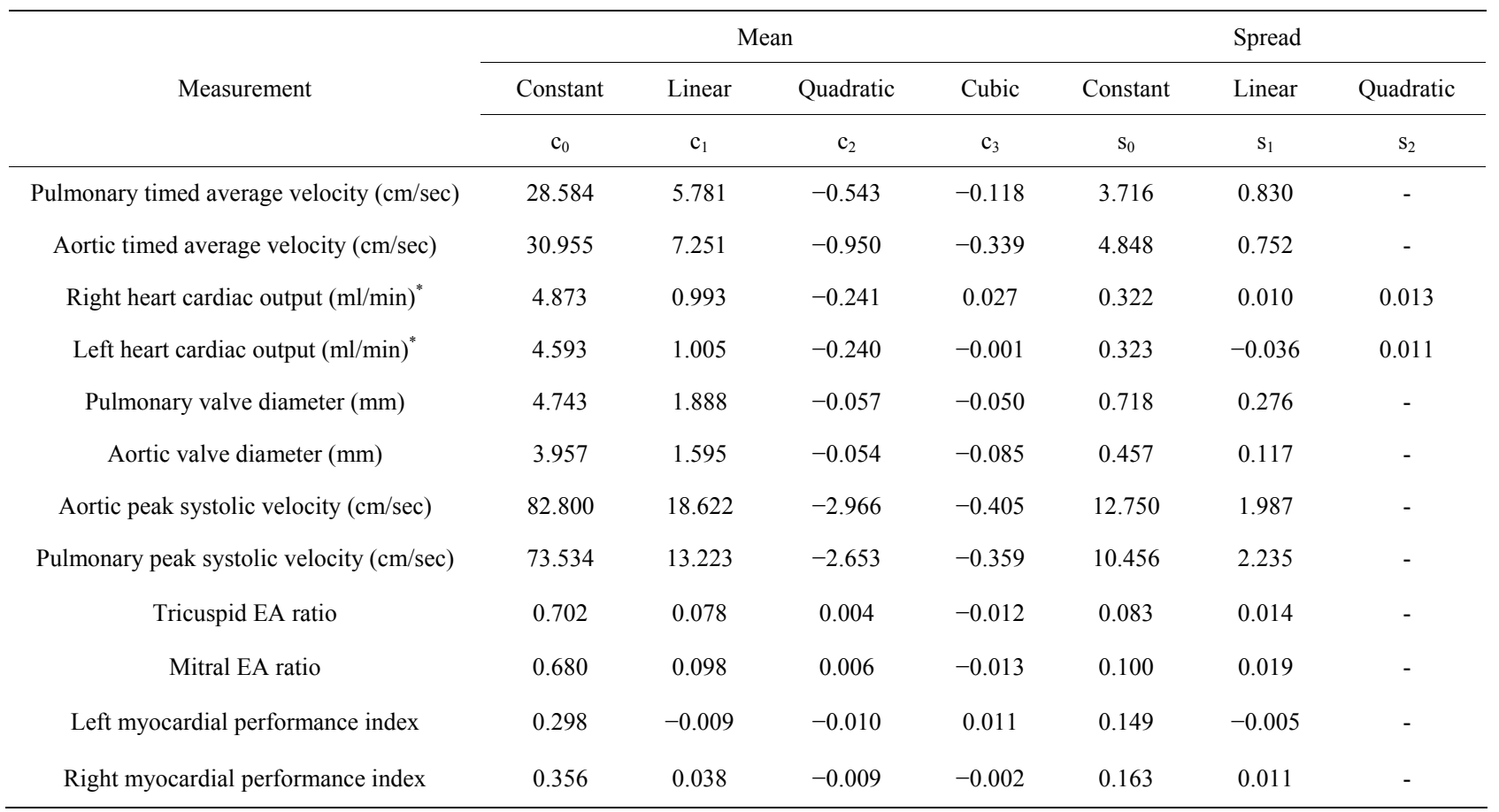

The percentile $(\mathrm{p})$ point is then given by mean + $[$ spread $\times \Phi(\mathrm{p})]$ where $\Phi$ is the standard normal cumulative distribution function. Studying eight subjects per week of gestation from 12 to 39 weeks and using our methods we estimate that the standard error for estimateing centiles is 0.063 standard deviation units in the middle $60 \%$ of the data, and 0.087 standard deviation units in the extreme high and low fifths.

We demonstrated that the cardiac output, peak systolic and time-averaged velocity of the outlets of both the right and left sides of the heart increase with advancing gestation. However the MPI showed little change across gestation. The E/A ratio on both sides of the heart increased with advancing gestation due to a rise in the amplitude of the E wave.

Using our method some measurements of MPI obtained were negative. This is inherent in our measurement method because the caliper lines used on the Doppler image to measure timings of inflow and outflow have some thickness. On the outflow Doppler we place the lines so that the inner border is touching the systolic 
flow curve (Figure 2). When measuring the interval between E/A complexes the lines are placed so that the outer border of the line is touching the complex (Figure 1). Thus outflow measurements are from inner border to inner border of the lines. Inflow measurements are from outer border to outer border. Where the inflow and outflow times are very similar this discrepancy can result in a negative value of MPI. Since this is an inherent result of the method we used we have included these values in the calculation of centile curves as their removal would introduce bias.

The mean gestational age and birth weight at delivery are summarised in Table 2. Using customised centile charts we found that $1.4 \%(\mathrm{n}=3)$ of babies had a birth weight of $<3^{\text {th }}$ centile and $3.2 \%(n=7)$ had a birth weight $>97^{\text {th }}$ centile. These women have been scanned at various gestations, potentially weeks before the birth of the baby which could explain the range of birth weights. As previously mentioned, we only excluded the women who developed complications of pregnancy prior to their scan and hence we included all the fetuses in the study as the growth at the time when the Doppler measurements were taken was within the normal range.

\section{DISCUSSION}

We have demonstrated that with modern equipment it is possible to use Doppler ultrasound to assess fetal cardiac function from twelve weeks of pregnancy to term using measures that provide information about diastolic, systolic and global cardiac function.

Gestation-specific ranges of left and right heart cardiac output from 13 to 41 weeks has been reported [8]. This provides a measure of systolic function. They found that the calculated 50 th centile for biventricular output increased from $40 \mathrm{ml} / \mathrm{min}$ at 15 weeks to $1470 \mathrm{ml} / \mathrm{min}$ at 40 weeks of gestation. Kiserud et al. calculated the mean fetal combined cardiac output to be $80 \mathrm{ml} / \mathrm{min}$ at 18 weeks and $1370 \mathrm{ml} / \mathrm{min}$ at 40 weeks in a cross-sectional study of 212 low-risk pregnancies with a gestational age of 18 to 41 weeks [10]. Arduini et al. have also described an exponential increase of the combined cardiac output from $120 \mathrm{ml} / \mathrm{min}$ at 15 weeks to $1800 \mathrm{ml} / \mathrm{min}$ at term [11]. However, the formula reported by Arduini et al. to calculate the time velocity integral (TVI) is incorrect. They report calculating the TVI $(\mathrm{cm})$ as mean velocity $(\mathrm{cm} / \mathrm{sec})$ divided by the duration of the cardiac cycle (sec) whereas it is a product of the two components. We calculated the combined output at 15 weeks of gestation

Table 2. Pregnancy outcome.

\begin{tabular}{ccc}
\hline & Mean & Range \\
\hline Gestation at delivery (weeks) & 39.5 & $30-40$ \\
Birth weight (grams) & 3456.25 & $1480-5260$ \\
\hline
\end{tabular}

to be $32 \mathrm{ml} / \mathrm{min}$, which increased to $727 \mathrm{ml} / \mathrm{min}$ at term. The ratio of the right to left cardiac output has been shown to be 1.3 by Arduini et al. whereas we described the ratio to be 1.2 at 15 weeks and 1.6 at 39 weeks. We found a lower cardiac output at term than in previous studies, but neither Arduini et al. nor Mielke and Benda provide separate details of the valve diameters or the average velocity of flow, so we cannot determine why our values differ. However our outlet valve diameters are similar to the values described by Kiserud et al. and hence we assume that the differences in cardiac output are due to a difference in the calculation of time velocity integral, presumably due to lower measurements of the time averaged velocity of flow.

Diastolic function, which provides information about the compliance of cardiac muscle and its ability to relax, can be assessed by the flow patterns through the tricuspid and mitral valves in diastole, and global cardiac function can be assessed by the MPI.

The change in tricuspid and mitral E and A wave velocities has been described from 17 weeks until term, but not earlier [12]. They reported that the E/A ratios of both the mitral and tricuspid valves increased with gestation and the changes were mainly due to an increase in the $\mathrm{E}$ wave as the A wave remains constant through gestation. They found that the tricuspid velocities were greater than the mitral velocities. In the present study, we also demonstrated an increase in E/A ratios of both valves with advancing gestation. The tricuspid $\mathrm{E} / \mathrm{A}$ ratios were calculated as 0.68 at 15 weeks and 0.82 at 39 weeks. The mitral $\mathrm{E} / \mathrm{A}$ ratios are 0.64 at 15 weeks and 0.87 at 39 weeks. The rise in the E/A ratios was due to an increase in the $\mathrm{E}$ wave with advancing gestation.

Friedman et al. evaluated the left ventricular myocardial performance index by placing the Doppler gate at the junction of the anterior mitral leaflet and the left outflow tract to identify simultaneously movement of the valve leaflets of the mitral and aortic valves [9]. They reported that their method was more reproducible than relying on Doppler measurement of blood flow. However, this method cannot be used for the right heart, where the pulmonary and tricuspid valves are physically widely separated, nor is it suitable for use in early pregnancy so we chose, instead, to perform the measurements on Doppler waveforms which were obtained separately for the outlet and inlet valves. This allowed us to establish gestation-specific ranges for both the left and right heart in the same fashion from 12 weeks to term.

Tsutsumi et al. reported the left ventricular MPI to be $0.62 \pm 0.07$ between $18-26$ weeks of gestation [13] They also reported a decrease to $0.43 \pm 0.03$ after 34 weeks and attributed the fall to developmental changes within the myocardium with increasing gestation. Mori et al. evaluated the MPI in fetuses with ductal constricttion [14]. They found that the right MPI increased in 
those with ductal constriction whereas the left MPI was unaffected. They did not find any change of MPI with gestation in normal fetuses and reported the right MPI to be $0.35 \pm 0.07$ and left MPI to be $0.35 \pm 0.03$.

Eidem et al. compared the MPI in fetuses between 20 40 weeks of gestation with a group of normal children aged 3 - 18 yrs [15]. They reported the fetal left MPI as $0.36 \pm 0.06$ and the right MPI as $0.35 \pm 0.05$ which was not statistically different from postnatal measurements in the children. We found the left ventricular MPI to be $0.29 \pm 0.15$ and the right ventricular MPI to be $0.34 \pm$ 0.16 between 12 - 39 weeks of gestation. Using a different technique, relying on valve movements rather than Doppler flow, Friedman et al. evaluated the left ventricular MPI between 18 and 31 weeks and found a higher mean value of $0.53 \pm 0.13$ that was independent of gestational age in their group [9].

Two studies in diabetic mothers suggest that examination of these indices can demonstrate significant changes in fetal heart function, thus suggesting that these methods are suitable for examination of physiological changes that may predispose to adult disease. Weiner et al. found differences in late pregnancy in E/A ratios in the fetuses of diabetic mothers compared with controls [16]. Russell described changes in the MPI at 12 weeks in the fetuses of women with diabetes, which were no longer present by late pregnancy, although there was then a demonstrable increase in the thickness of the ventricular septum [17]. This suggests that changes in cardiac function in early pregnancy that can be assessed with Doppler, may pre-date structural changes in the heart that become apparent in late pregnancy.

In conclusion, fetal cardiac physiology can be studied and Doppler indices reliably measured as early as the late first trimester of pregnancy and study of this may eventually provide better understanding of changes which may predispose to adult cardiac disease.

\section{REFERENCES}

[1] Tei, C., Dujardin, K., Hodge, D., et al. (1996) Doppler index combining systolic and diastolic myocardial performance: Clinical value in cardiac amyloidosis. Journal of the American College of Cardiology, 28, 658-664.

[2] Williams, R., Ritter, S., Tani, L.Y., et al. (2000) Quantitative assessment of ventricular function in children with single ventricle using the Doppler myocardial performance index. American Journal of Cardiology, 86, 11061110. doi:10.1016/S0002-9149(00)01168-1

[3] Ichizuka, K., Matsuoka, R., Hasegawa, J., et al. (2005) The Tei index for evaluation of fetal myocardial performance in sick fetuses. Early Human Development, 81, 273-279. doi:10.1016/j.earlhumdev.2004.07.003

[4] Crispi, F., Hernandez-Andrade, E., Pelsers, M.M.A.L., et al. (2008) Cardiac dysfunction and cell damage across clinical stages of severity in growth restricted fetuses.
American Journal of Obstetrics \& Gynecology, 199, 254.e1-254.e8.

[5] Hanson, M.A. and Gluckman, P.D. (2005) Developmental processes and the induction of cardiovascular function: Conceptual aspects. The Journal of Physiology, 565, 2734. doi:10.1113/jphysiol.2004.082339

[6] Davis, L., Thornburg, K.L. and Giraud, G.D. (2005) The effects of anaemia as a programming agent in the fetal heart. The Journal of Physiology, 565, 35-41. doi:10.1113/jphysiol.2004.082388

[7] Battista, M.C., Calvo, E., Chorvatova, A., et al. (2005) Intra-uterine growth restriction and the programming of left ventricular remodelling in female rats. The Journal of Physiology, 565, 197-205.

doi:10.1113/jphysiol.2004.078139

[8] Mielke, G. and Benda, N. (2001) Cardiac output and central distribution of blood flow in the human fetus. Circulation, 103, 1662-1668. doi:10.1161/01.CIR.103.12.1662

[9] Friedman, D., Buyon, J., Kim, M., et al. (2003) Fetal cardiac function assessed by Doppler myocardial performance index (Tei index). Ultrasound in Obstetrics \& Gynecology, 21, 33-36 doi:10.1002/uog.11

[10] Kiserud, T., Ebbing, C., Kessler, J., et al. (2006) Fetal cardiac output, distribution to the placenta and impact of placental compromise. Ultrasound in Obstetrics \& Gynecology, 28, 126-136 doi:10.1002/uog.2832

[11] Arduini, D., Rizzo, G., Romanini, C., et al. (1995) Fetal cardiac output measurement in normal and pathologic states. In: Copel, J.A. and Reed, K.L., Doppler Ultrasound in Obstetrics and Gynecology, Springer Publishing Company, New York, Chap 27.

[12] Fernandez Pineda, L., Tamariz-Martel Moreno, A., Maitre Azcarate, M.J., et al. (2000) Contribution of Doppler atrioventricular flow waves to ventricular filling in the human fetus. Pediatric Cardiology, 21, 422-428. doi:10.1007/s002460010101

[13] Tsutsumi, T., Ishii, M., Eto, G., et al. (1999) Serial evaluation for myocardial performance in fetuses and neonates using a new Doppler Index. Pediatrics International, 41, 722-727. doi:10.1046/j.1442-200x.1999.01155.x

[14] Mori, Y., Rice, M.J., McDonald, R.W., et al. (2001) Evaluation of systolic and diastolic ventricular performance of the right ventricle in fetuses with ductal constricttion using the Doppler Tei index. American Journal of Cardiology, 88, 1173-1178. doi:10.1016/S0002-9149(01)02056-2

[15] Eidem, B.W., Edwards, J.M. and Cetta, F. (2001) Quantitative assessment of fetal ventricular function: Establishing normal values of the myocardial performance index in the fetus. Echocardiography, 18, 9-13. doi:10.1046/j.1540-8175.2001.t01-1-00009.x

[16] Weiner, Z., Zloczower, M., Lerner, A., et al. (1999) Cardiac compliance in fetuses of diabetic women. Obstetrics \& Gynecology, 93, 948-951. doi:10.1016/S0029-7844(99)00003-4

[17] Russell, N., Foley, M., Kinsley, B., et al. (2007) Fetal cardiac function in type 1 diabetic pregnancy. Journal of Obstetrics and Gynaecology, 27, S14. 